hep-ph/0209295

September 2002

\title{
Evaluating double and triple (?) boxes
}

\author{
V.A. Smirnova因 \\ a Nuclear Physics Institute of Moscow State University, \\ Moscow 119992, Russia
}

A brief review of recent results on analytical evaluation of double-box Feynman integrals is presented. First steps towards evaluation of massless on-shell triple-box Feynman integrals within dimensional regularization are described. The leading power asymptotic behaviour of the dimensionally regularized massless on-shell master planar triple-box diagram in the Regge limit $t / s \rightarrow 0$ is evaluated. The evaluation of the unexpanded master planar triple box is outlined and explicit results for coefficients at $1 / \epsilon^{j}, \mathrm{j}=2, \ldots, 6$, are presented.

\section{Double boxes}

Feynman diagrams with four external lines contribute to many important physical quantities. They are rather complicated mathematical objects because they depend on many variables: internal masses, Mandelstam variables and squares of external momenta. The most complicated twoloop diagrams are the planar double box and nonplanar (crossed) double box. Almost all available analytical results correspond to the massless diagrams. Ironically, the first result for the massless double boxes was obtained in the most complicated case, where all the fours external legs are off-shell, i.e. $p_{i}^{2} \neq 0$ for all $i=1,2,3,4$. This is an elegant analytical result for the scalar planar master double box (and, moreover, for a general planar ladder diagram of this type), i.e. for all powers of propagators equal to one, obtained in [1]. However, no other results for the pure offshell double boxes (e.g. with dots on some lines and/or with some lines, other than rungs, contracted) have been derived up to now so that this result stays unique in the pure off-shell category.

For massless double-box diagrams with at least

\footnotetext{
*Talk presented at the International Symposium Radcor 2002 and Loops and Legs 2002 (September 8-13, Kloster Banz, Germany). Supported by INTAS through grant 0000313, the Russian Foundation for Basic Research through project 01-02-16171, and the Volkswagen Foundation, contract No. I/77788.
}

one leg on the mass shell, i.e. $p_{i}^{2}=0$, infrared and collinear divergences appear, so that one introduces a regularization which is usually chosen to be dimensional [2], with the space-time dimension $d$ as a regularization parameter. One hardly believes that a regularized double-box diagram can be analytically evaluated for the general value of the regularization parameter $\epsilon=(4-d) / 2$, and the evaluation is usually performed in a Laurent expansion in $\epsilon$, typically, up to a finite part.

The problem of the evaluation of Feynman integrals associated with a given graph according to some Feynman rules is usually decomposed into two parts: reduction of general Feynman integrals of this class to so-called master integrals (which cannot be simplified further) and the evaluation of these master integrals. A standard tool to solve the first part of this problem is the method of integration by parts (IBP) [ 3] when one writes down identities obtained by putting to zero various integrals of derivatives of the general integrand connected with the given graph and tries to solve a resulting system of equations to obtain recurrence relations that express Feynman integrals with general integer powers of the propagators through the master integrals.

The most complicated basic master planar and non-planar on-shell massless double-box diagram were calculated in [ 4, 5] by a method based on Feynman parameters and Mellin-Barnes (MB) 
representation (see [6] for details of the method). It turns out that it is natural to consider nonplanar double boxes as functions of the three Mandelstam variables $s, t$ and $u$ not necessarily restricted by the physical condition $s+t+u=0$ which does not simplify the result.

Reduction procedures for the evaluation of general double-box diagrams, with arbitrary numerators and integer powers of the propagators were developed in [ 7] in the planar case and in [ 8] in the non-planar case. In [ 7], the first of the two most complicated master integrals involved is with all powers of propagators equal to one. As a second complicated master integral, the authors of [7] have chosen the diagram with a dot on the central line. As was pointed out later [ 9], in practical calculations one runs into a linear combination of these two master integrals with the coefficient $1 / \epsilon$, so that a problem has arisen because the calculation of the master integrals in one more order in $\epsilon$ looked rather nasty. Two solutions of this problem immediately appeared. In [10], the authors calculated this very combination of the master integrals, while in [11] another choice of the master integrals was made: as a second complicated master integral, the authors have taken the integral which is obtained from the first master integral by inserting a specific numerator. This was a more successful choice because, according to the calculational experience, no negative powers of $\epsilon$ occur as coefficients at these two master integrals.

These analytical algorithms were successfully applied to the evaluation of two-loop virtual corrections to various scattering processes [ 12] in the zero-mass approximation.

In the case, where one of the external legs is on-shell, $p_{1}^{2} \neq 0, p_{i}^{2}=0, i=2,3,4$, the planar double box and one of two possible non-planar double-box diagrams with all powers of propagators equal to one were analytically calculated in [13], as functions of the Mandelstam variables $s$ and $t$ and the non-zero external momentum squared $p_{1}^{2}$. Explicit results were expressed through (generalized) polylogarithms, up to the fourth order, dependent on rational combinations of $p_{1}^{2}, s$ and $t$, and a one- and (in the non-planar case) two-dimensional integrals with simple inte- grands. To do this, the method based on MB integrals mentioned above was applied. These and other master planar and non-planar double boxes with one leg off-shell were evaluated in 14 with the help of the method of differential equations [ 15]. The corresponding results are expressed through so-called two-dimensional harmonic polylogarithms which generalize harmonic polylogarithms [16].

A reduction procedure that provides the possibility to express any given Feynman integral to the master integrals was also developed in [14]. It is based on the Laporta's observation that, when increasing the total dimension of the denominator and numerator in Feynman integrals associated with the given graph, the total number of IBP and Lorentz-invariance equations grows faster than the number of independent Feynman integrals. These techniques were successfully applied [ 17] to the Feynman integrals with one leg off-shell contributing to the process $e^{+} e^{-} \rightarrow 3$ jets.

For another three-scale calculational problem, where all four legs are on-shell and there is a non-zero internal mass, a first analytical result was obtained in [18 for the scalar master double box. These and other future similar results will be used for calculations connected with Bhabha scattering (see also [19] for some steps in this direction).

It is believed that sooner or later we shall achieve the limit in the process of analytical evaluation of Feynman integrals so that we shall be forced to proceed only numerically. (See, e.g., [20] where this point of view has been emphasized.) However the dramatic recent progress in the field of analytical evaluation of Feynman integrals shows that we have not yet exhausted our abilities in this direction. Indeed, several powerful methods were developed last years. To calculate the master integrals one can apply the technique of $\mathrm{MB}$ integration and the method of differential equations mentioned above. To construct appropriate recursive algorithms one can use recently developed methods based on shifting dimension [21] and differential equations [ 14] as well as a method based on non-recursive solutions of recurrence relations [22.

One can also hope that new analytical re- 
sults can be obtained for many other classes of Feynman integrals depending on two and three scales. In particular, the analytical evaluation of any two-loop two-scale Feynman integral with two, three and four legs looks quite possible. In fact, when going to a higher level of calculational complexity, one increases the number of loops, legs and independent variables. As the experience with the double boxes with one leg offshell has shown, the crucial point is to introduce an appropriate class of functions. In this example, these are two-dimensional harmonic polylogarithms which turn out to be adequate functions to express result for diagrams of the given family. Presumably, when turning to a situation with one more kinematical invariant, e.g. when one more leg is off-shell, a natural procedure will be to introduce three-dimensional harmonic polylogarithms, etc.

However, when the number of loops is increased one does not need to introduce new functions, so that this transition means a 'pure' calculational complication.

\section{Triple boxes}

Let us now turn our attention to three-loop onshell massless four-point diagrams and consider dimensionally regularized massless on-shell planar triple box diagram shown in Fig. 1. The gen-

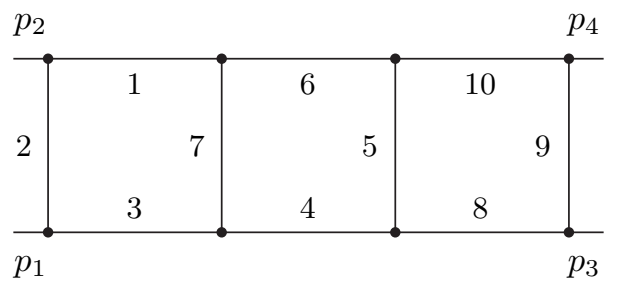

Figure 1. Planar triple box diagram.

eral planar triple box Feynman integral without numerator takes the form

$T\left(a_{1}, \ldots, a_{10} ; s, t ; \epsilon\right)=\iiint \frac{\mathrm{d}^{d} k \mathrm{~d}^{d} l \mathrm{~d}^{d} r}{\left(k^{2}\right)^{a_{1}}}$

$$
\begin{aligned}
& \times \frac{1}{\left[\left(k+p_{2}\right)^{2}\right]^{a_{2}}\left[\left(k+p_{1}+p_{2}\right)^{2}\right]^{a_{3}}} \\
& \times \frac{1}{\left[\left(l+p_{1}+p_{2}\right)^{2}\right]^{a_{4}}\left[(r-l)^{2}\right]^{a_{5}}\left(l^{2}\right)^{a_{6}}} \\
& \times \frac{1}{\left[(k-l)^{2}\right]^{a_{7}\left[\left(r+p_{1}+p_{2}\right)^{2}\right]^{a_{8}}}} \\
& \times \frac{1}{\left[\left(r+p_{1}+p_{2}+p_{3}\right)^{2}\right]^{a_{9}}\left(r^{2}\right)^{a_{10}}},
\end{aligned}
$$

where $s=\left(p_{1}+p_{2}\right)^{2}$ and $t=\left(p_{2}+p_{3}\right)^{2}$ are Mandelstam variables, and $k, l$ and $r$ are loop momenta. Usual prescriptions $k^{2}=k^{2}+i 0, s=$ $s+i 0$, etc. are implied.

By a straightforward generalization of two-loop manipulations, one arrives [23] at a sevenfold MB representation of (1). In the case of the master triple box, $a_{i}=1, i=1,2, \ldots, 10$, we have

$$
\begin{aligned}
& T^{(0)}(s, t ; \epsilon) \equiv T(1, \ldots, 1 ; s, t ; \epsilon) \\
& =\frac{\left(i \pi^{d / 2}\right)^{3}}{\Gamma(-2 \epsilon)(-s)^{4+3 \epsilon} \frac{1}{(2 \pi i)^{7}}} \\
& \times \int_{-i \infty}^{+i \infty} \mathrm{d} w \prod_{j=2}^{7} \mathrm{~d} z_{j}\left(\frac{t}{s}\right)^{w} \frac{\Gamma(1+w) \Gamma(-w)}{\Gamma\left(1-2 \epsilon+w-z_{4}\right)} \\
& \times \frac{\Gamma\left(-\epsilon+z_{2}\right) \Gamma\left(-\epsilon+z_{3}\right) \Gamma\left(1+w-z_{4}\right) \Gamma\left(-z_{5}\right)}{\Gamma\left(1+z_{2}+z_{4}\right) \Gamma\left(1+z_{3}+z_{4}\right)} \\
& \times \frac{\Gamma\left(1+\epsilon+z_{4}\right) \Gamma\left(z_{2}+z_{4}\right) \Gamma\left(z_{3}+z_{4}\right) \Gamma\left(-z_{6}\right)}{\Gamma\left(1-z_{5}\right) \Gamma\left(1-z_{6}\right)} \\
& \times \frac{\Gamma\left(w+z_{2}+z_{3}+z_{4}-z_{7}\right) \Gamma\left(-z_{2}-z_{3}-z_{4}\right)}{\Gamma\left(1-2 \epsilon+z_{5}+z_{6}+z_{7}\right)} \\
& \times \Gamma\left(1+\epsilon+w-z_{4}-z_{5}-z_{6}-z_{7}\right) \Gamma\left(1+z_{7}\right) \\
& \times \Gamma\left(-1-\epsilon-z_{5}-z_{7}\right) \Gamma\left(-1-\epsilon-z_{6}-z_{7}\right) \\
& \times \Gamma\left(1+z_{5}+z_{6}+z_{7}\right) \Gamma\left(-\epsilon-w-z_{2}+z_{5}+z_{7}\right) \\
& \times \Gamma\left(2+\epsilon+z_{5}+z_{6}+z_{7}\right) \\
& \times \Gamma\left(-\epsilon-w-z_{3}+z_{6}+z_{7}\right) .
\end{aligned}
$$

As a first step of three-loop calculations, let us consider the evaluation [23 of the leading power asymptotic behaviour of (2) in the Regge limit $t / s \rightarrow 0$ This calculation demonstrates that a three-loop BFKL [24] analysis is possible.

One can use the strategy of expansion by regions [25, 26, 27] which shows that in the leading power only $(1 \mathrm{c}-1 \mathrm{c}-1 \mathrm{c})$ and $(2 \mathrm{c}-2 \mathrm{c}-2 \mathrm{c})$ regions contribute, with the leading power $1 / t$. (See [26] and Chapter 8 of [27] for definitions of these contribu- 
tions.) The leading power $(2 \mathrm{c}-2 \mathrm{c}-2 \mathrm{c})$ contribution for the master planar triple box takes the form

$$
\begin{aligned}
& T^{(0),(2 c-2 c-2 c)}(s, t ; \epsilon)=\iiint \frac{\mathrm{d}^{d} k \mathrm{~d}^{d} l \mathrm{~d}^{d} r}{k^{2}\left(k+p_{2}\right)^{2}} \\
& \quad \times \frac{1}{\left(2 p_{1} k+s\right)\left(2 p_{1} l+s\right)(r-l)^{2} l^{2}(k-l)^{2}} \\
& \quad \times \frac{1}{\left(2 p_{1} r+s\right)\left(r+p_{2}+\tilde{p}\right)^{2} r^{2}},
\end{aligned}
$$

where $\tilde{p}$ is such that $\tilde{p}^{2}=t, 2 p_{1} \tilde{p}=0,2 p_{2} \tilde{p}=$ $-t$. The leading power (1c-1c-1c) contribution is obtained due to the symmetry $\{1 \leftrightarrow 3,4 \leftrightarrow$ $6,8 \leftrightarrow 10\}$.

On the other hand, one can organize the calculational procedure in such a way that the calculation of the Regge asymptotics is a part of the calculation of the unexpanded triple box. An analysis of the integrand shows that the key gamma functions that are responsible for the leading Regge behaviour are $\Gamma\left(-\epsilon+z_{2,3}\right)$ and $\Gamma\left(-1-\epsilon-z_{6,5}-z_{7}\right)$. The standard procedure of shifting contours and taking residues can be applied. It results again in a sum of MB integrals where a Laurent expansion of the integrand in $\epsilon$ is possible. The final result for the Regge asymptotics of the planar triple box is [23]:

$$
\begin{aligned}
& T^{(0)}(s,t \epsilon)=-\frac{\left(i \pi^{d / 2} \mathrm{e}^{-\gamma_{\mathrm{E}} \epsilon}\right)^{3}}{s^{3}(-t)^{1+3 \epsilon}} \\
& \times\left\{\frac{16}{9 \epsilon^{6}}-\frac{5 L}{3 \epsilon^{5}}-\frac{3 \pi^{2}}{2 \epsilon^{4}}-\left[\frac{11 \pi^{2}}{12} L+\frac{131 \zeta(3)}{9}\right] \frac{1}{\epsilon^{3}}\right. \\
&+\left[\frac{49 \zeta(3)}{3} L-\frac{1411 \pi^{4}}{1080}\right] \frac{1}{\epsilon^{2}} \\
&-\left[\frac{503 \pi^{4}}{1440} L-\frac{73 \pi^{2} \zeta(3)}{4}+\frac{301 \zeta(5)}{15}\right] \frac{1}{\epsilon} \\
&+\left[\frac{223 \pi^{2} \zeta(3)}{12}+149 \zeta(5)\right] L \\
&\left.-\frac{624607 \pi^{6}}{544320}+\frac{167 \zeta(3)^{2}}{9}+O(\epsilon)\right\}
\end{aligned}
$$

where $L=\ln s / t, \gamma_{\mathrm{E}}$ is the Euler constant and $\zeta(z)$ is the Riemann zeta function.

For the analytic evaluation of (2), without expansion, a similar procedure can be applied.
Eventually, we arrive at

$T^{(0)}(s, t ; \epsilon)=-\frac{\left(i \pi^{d / 2} \mathrm{e}^{-\gamma_{\mathrm{E}} \epsilon}\right)^{3}}{s^{3}(-t)^{1+3 \epsilon}} \sum_{i=0}^{6} \frac{c_{j}(x, L)}{\epsilon^{j}}$,

where, up to $1 / \epsilon^{2}$ terms, we have

$$
\begin{aligned}
c_{6}= & \frac{16}{9}, \quad c_{5}=-\frac{5 L}{3}, \quad c_{4}=-\frac{3 \pi^{2}}{2}, \\
c_{3}= & 3\left(\operatorname{Li}_{3}(-x)+L \operatorname{Li}_{2}(-x)\right) \\
& -\frac{3}{2}\left(L^{2}+\pi^{2}\right) \ln (1+x)-\frac{11}{12} L \pi^{2}-\frac{131}{9} \zeta(3), \\
c_{2}= & 3\left(S_{2,2}(-x)+L S_{1,2}(-x)\right)-51 \operatorname{Li}_{4}(-x) \\
& +3 \ln (1+x)\left(\operatorname{Li}_{3}(-x)+L \operatorname{Li}_{2}(-x)\right) \\
& -37 L \operatorname{Li}_{3}(-x)-\frac{23}{2} L^{2} \operatorname{Li}_{2}(-x) \\
& -8 \pi^{2} \operatorname{Li}_{2}(-x)-\frac{3}{4}\left(L^{2}+\pi^{2}\right) \ln ^{2}(1+x) \\
& -3 \ln (1+x) \zeta(3)+\frac{3}{2} L^{3} \ln (1+x) \\
& +L \pi^{2} \ln (1+x)-\frac{1411}{1080} \pi^{4}+\frac{49}{3} L \zeta(3) .
\end{aligned}
$$

Here $x=t / s$, so that $L=-\ln x$, and

$S_{a, b}(z)=\frac{(-1)^{a+b-1}}{(a-1) ! b !} \int_{0}^{1} \frac{\ln ^{a-1}(t) \ln ^{b}(1-z t)}{t} \mathrm{~d} t$

are the generalized polylogarithms [ 28].

The coefficient functions $c_{i}, i=2, \ldots, 6$, have been confirmed [29] by a numerical check with the help of numerical integration in the space of alpha parameters [ 30]. (This algorithm is based on a procedure of resolution of singularities in the alpha representation that was used in early papers on renormalization theory (see, e.g., [ 32]) and in proofs of similar results for asymptotic expansions of Feynman integrals in limits of momenta and masses (see, e.g., [33] and Appendix B of $[27])$.

The present status of the calculation is as follows. After integrating over some of the variables $z_{i}, c_{1}$ and $c_{0}$ are expressed through twodimensional MB integrals. In some of them, the last integration over a $z$-variable can be performed and resulting integrals over $w$ can be evaluated by closing the integration contour to the right and taking residues at $w=0,1,2, \ldots$. The corresponding results are expressed in terms of 
harmonic polylogarithms [31] with parameters 0 and 1 . In the rest of the contributions to $c_{1}$ and $c_{0}$, subintegrations cannot be done due to Barnes lemmas. Hopefully, such two-dimensional MB integrals can be analytically evaluated by means of 'experimental mathematics' similar to used in [ 18, with results presumably expressed in terms of harmonic polylogarithms.

The procedure described above can be applied, in a similar way, to the calculation of any massless planar on-shell triple box.

Acknowledgments. I am grateful to V.S. Fadin and A.A. Penin for helpful discussions of perspectives of the three-loop BFKL analysis. Thanks again to G. Heinrich for numerical checks.

\section{REFERENCES}

[1] N.I. Ussyukina and A.I. Davydychev, Phys. Lett. B298 (1993) 363; B305 (1993) 136.

[2] G. 't Hooft and M. Veltman, Nucl. Phys. B44 (1972) 189; C.G. Bollini and J.J. Giambiagi, Nuovo Cim. 12B (1972) 20.

[3] K.G. Chetyrkin and F.V. Tkachov, Nucl. Phys. B192 (1981) 159.

[4] V.A. Smirnov, Phys. Lett. B460 (1999) 397.

[5] J.B. Tausk, Phys. Lett. B469 (1999) 225.

[6] V.A. Smirnov, hep-ph/0209177.

[7] V.A. Smirnov and O.L. Veretin, Nucl. Phys. B566 (2000) 469.

[8] C. Anastasiou, T. Gehrmann, C. Oleari, E. Remiddi and J.B. Tausk, Nucl. Phys. B580 (2000) 577;

[9] E.W.N. Glover and M.E. Tejeda-Yeomans, Nucl. Phys. Proc. Suppl. 89 (2000) 196.

[10] T. Gehrmann and E. Remiddi, Nucl. Phys. Proc. Suppl. 89 (2000) 251.

[11] C. Anastasiou, J.B. Tausk and M.E. TejedaYeomans, Nucl. Phys. Proc. Suppl. 89 (2000) 262.

[12] Z. Bern, L. Dixon and A. Ghinculov, Phys. Rev. D63 (2001) 053007; C. Anastasiou, E.W.N. Glover, C. Oleari and M.E. TejedaYeomans, Nucl. Phys. B601 (2001) 318; B601 (2001) 341; Nucl. Phys. B605 (2001) 486; E.W.N. Glover, C. Oleari and M.E. TejedaYeomans, Nucl. Phys. B605 (2001) 467; Z.
Bern, A. De Freitas and L.J. Dixon, JHEP 0109 (2001) 037; Z. Bern, A. De Freitas, L.J. Dixon, A. Ghinculov and H.L. Wong, JHEP 0111 (2001) 031; Z. Bern, A. De Freitas and L.J. Dixon, JHEP 0203 (2002) 018; C. Anastasiou, E.W.N. Glover and M.E. TejedaYeomans, Nucl. Phys. B629 (2002) 255; E.W.N. Glover, talk at Radcor/Loops\&Legs 2002.

[13] V.A. Smirnov, Phys. Lett. B491 (2000) 130; B500 (2001) 330.

[14]T. Gehrmann and E. Remiddi, Nucl. Phys. B601 (2001) 248; B601 (2001) 287.

[15] A.V. Kotikov, Phys. Lett. B254 (1991) 158; B259 (1991) 314; B267 (1991) 123; E. Remiddi, Nuovo Cim. 110A (1997) 1435; T. Gehrmann and E. Remiddi, Nucl. Phys. B580 (2000) 485.

[16] E. Remiddi and J.A.M. Vermaseren, Int. J. Mod. Phys. A15 (2000) 725.

[17] L.W. Garland, T. Gehrmann, E.W.N. Glover, A. Koukoutsakis and E. Remiddi, Nucl. Phys. B627 (2002) 107; hep-ph/0206067; S. Moch, P. Uwer and S. Weinzierl, hep-ph/0110407; T. Gehrmann, talk at Radcor/Loops\&Legs 2002; P. Uwer, talk at Radcor/Loops\&Legs 2002.

[18] V.A. Smirnov, Phys. Lett. B524 (2002) 129.

[19] A. Werthenbach, talk at Radcor/Loops\&Legs 2002; J. Fleischer, talk at Radcor/Loops\&Legs 2002.

[20] G. Passarino, Nucl. Phys. B619 (2001) 257.

[21] O.V. Tarasov, Nucl. Phys. B480 (1996) 397; Phys. Rev. D54 (1996) 6479; talk at Radcor/Loops\&Legs 2002.

[22] P.A. Baikov, Phys. Lett. B385 (1996) 404; Nucl. Instrum. Methods A389 (1997) 347; Phys. Lett. B474 (2000) 385; talk at Radcor/Loops\&Legs 2002.

[23] V.A. Smirnov, hep-ph/0209193.

[24] L.N. Lipatov, Sov. J. Nucl. Phys. 23 (1976) 642; V.S. Fadin, E.A. Kuraev and L.N. Lipatov, Phys. Lett. B60 (1975) 50; E.A. Kuraev, L.N. Lipatov and V.S. Fadin, Sov. Phys. JETP 44 (1976) 45; 45 (1977) 199. Ya.Ya. Balitsky and L.N. Lipatov, Sov. J. Nucl. Phys. 28 (1978) 822.

[25] M. Beneke and V.A. Smirnov, Nucl. Phys. 
B522 (1998) 321.

[26] V.A. Smirnov and E.R. Rakhmetov, Teor. Mat. Fiz. 120 (1999) 64; V.A. Smirnov, Phys. Lett. B465 (1999) 226.

[27] V.A. Smirnov, Applied Asymptotic Expansions in Momenta and Masses (Springer, Berlin, Heidelberg, 2001).

[28] K.S. Kölbig, J.A. Mignaco and E. Remiddi, BIT 10 (1970) 38; K.S. Kölbig, Math. Comp. 39 (1982) 647; A. Devoto and D.W. Duke, Riv. Nuovo Cim. 7, No. 6 (1984) 1.

[29] G. Heinrich, private communication.

[30] T. Binoth and G. Heinrich, Nucl. Phys. B585 (2000) 741 .

[31] E. Remiddi and J.A.M. Vermaseren, Int. J. Mod. Phys. A15 (2000) 725.

[32] K. Hepp, Commun. Math. Phys. 2 (1966) 301.

[33] V.A. Smirnov, Commun. Math. Phys. 134 (1990) 109. 